\title{
Monte Carlo studies of slow relaxation in diluted antiferromagnets
}

\author{
U. Nowak and K. D. Usadel \\ Theoretische Physik and Sonderforschungsbereich No. 166, Universität-GH-Duisburg, Lotharstrasse 1, \\ 4100 Duisburg, Federal Republic of Germany
}

(Received 26 July 1988)

\begin{abstract}
Monte Carlo simulations of diluted Ising-type antiferromagnets in a uniform external magnetic field in three dimensions show rather strong irreversibilities in the magnetization and a slow relaxation of the remanent magnetization of the domain state. A detailed investigation of the domain state and its relaxation shows that for low temperatures domains are strongly pinned at vacancies and that relaxation primarily takes place in the domain walls.
\end{abstract}

\section{INTRODUCTION}

Recently, there have been several theoretical and experimental papers on the behavior of domains in random-field systems. ${ }^{1}$ Experiments are usually performed on diluted Ising-type antiferromagnets in a uniform external field (DAFF) which belong to the same universality class as the random-field Ising model (RFIM). ${ }^{2}$ However, only very recently it has been realized that the mapping of a DAFF system on a ferromagnet which gives rise not only to random fields but also to random bonds leads to important differences in both the crossover and the domain-state behavior of both systems. ${ }^{3,4}$

The domain state of the DAFF is obtained if the system is cooled in an external magnetic field from the high-temperature phase. From neutron scattering experiments it is concluded that this field-cooled (FC) state is frozen at low temperatures ${ }^{1}$ due to a strong domain-wall pinning in regions with many nonmagnetic ions ("vacancies"). This has been seen for the first time within mean field theory ${ }^{5-7}$ and also in Monte Carlo simulations. ${ }^{6}$

Quite recently, a very slow decay of the magnetization of the FC state after decreasing the magnetic field has been observed experimentally. ${ }^{8,9}$ In an attempt to explain the observed slow remanence relaxation Nattermann and Vilfan ${ }^{4}$ assumed that after removing the external field spin readjustments primarily take place only in the domain walls while the domain size does not change because of domain-wall pinning. Invoking scaling-type arguments a logarithmically slow temporal decay of the thermoremanent magnetization was obtained.

In order to further elucidate the mechanism for domain formation, pinning, and slow relaxation we have performed Monte Carlo simulations on DAFF in three dimensions. The simulations of the DAFF were performed on a simple-cubic lattice with linear size $N=60$. Throughout the dilution was $50 \%$, i.e., in between the percolation concentrations of the occupied sites and the empty sites, respectively. Periodic boundary conditions were employed. The spin configurations were updated using the Metropolis algorithm and the multispin coding technique was used. ${ }^{10}$ The simulations were performed on a Cyber 76 computer.
In the present paper we focus primarily on the relaxation behavior of the FC domain state (Sec. III). In addition, in the next section new results for the historydependent magnetization are presented.

\section{HISTORY-DEPENDENT MAGNETIZATION}

The domain state is reached through field cooling. The initial spin configuration is chosen at random corresponding to infinite temperature (paramagnetic phase). Then the system is cooled in a fixed field. As has been observed for the first time by Grest et al. ${ }^{6}$ within a Monte Carlo simulation the magnetization obtained in this way is larger at low temperatures than the magnetization obtained after zero field cooling (ZFC) and subsequent field heating $(\mathrm{FH})$. In the simulations the initial spin configuration for $\mathbf{F H}$ is chosen to be the ideal antiferromagnetic ordered state which is warmed up in a fixed magnetic field.

In our simulations we found a rather pronounced irreversibility in the FC-FH-curves at low temperatures in qualitative agreement with the Monte Carlo simulation of Ref. 6, and with recent experiments, ${ }^{8,9,11}$ see Fig. 1. In this simulation field cooling was started at $T=2.5$ and $H=1.0$ (all quantities are measured in units of the exchange interaction $J$ between a pair of neighboring Ising spins $( \pm 1)$ from an initial random spin configuration. The temperature was reduced in steps of $\delta T=0.1$. For comparison the Néel temperature (for $H=0$ ) is around 2.2 in these units. During the time $\delta t$ between two steps (corresponding to the inverse of the cooling rate) the magnetization was recorded. The FH curves were obtained in the same way starting at $T=0.1$ and $H=1.0$ from the antiferromagnetic ground state. Note that the splitting of the FC and FH curves is much larger than the splitting observed by Grest et al. ${ }^{6}$ The reason for this is the higher concentration of vacancies we used $150 \%$ while Grest et al. used only $30 \%$, i.e., a concentration just above the percolation threshold for a threedimensional simple-cubic lattice). Note that Grest et al. ${ }^{6}$ used different reduced units. To compare with our calculations their values of $H$ and $T$ have to be multiplied by 2 and 4 , respectively, in order to get our units.

As a new result we observed in our simulations that the FC-FH curves both are dependent on time: a reduction 


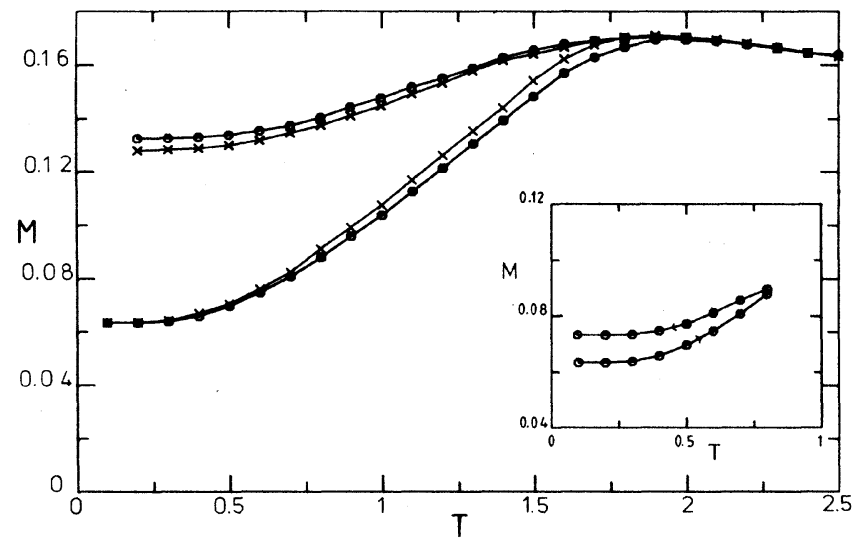

FIG. 1. Comparison of FC (upper curves) and FH (lower curves) magnetization for two different cooling-heating rates $(\delta t)^{-1}$. Circles: $\delta t=40 \mathrm{MCS} / \mathrm{spin}$; crosses: $\delta t=150 \mathrm{MCS} / \mathrm{spin}$. Inset shows magnetization during $\mathrm{FH}$ (lower curve) from the antiferromagnetic ground state to $T=0.8$ followed by field cooling (upper curve). $\delta t=40 \mathrm{MCS} / \mathrm{spin}$.

of the cooling rate leads to a reduction of the FC magnetization. Surprisingly, a corresponding effect upon heating is also observed for the FH magnetization. Results for two different cooling rates are shown in Fig. 1. In the inset of Fig. 1 is shown the magnetization during FH from the antiferromagnetic ground state to $T=0.8$, a temperature at which long-range order (LRO) is clearly present (see also Fig. 2). At this temperature the system was held for $\mathbf{4 0}$ Monte Carlo steps (MCS)/spin before it was cooled again. Note that during this waiting time the system relaxed to a state with slightly higher magnetization.

Since the FC state of the DAFF system for not too high magnetic fields is generally believed not to be the equilibrium state the observed dependence of the FC magnetization on cooling rate is what one would expect. On the other hand, the equilibrium state of the DAFF system in three dimensions should be ordered at low temperatures, ${ }^{12-14}$ which makes the observed dependence of the FH magnetization on cooling rate as well as the irreversibilities obtained when reversing the FH scan less obvious. The latter effect has also been observed experimentally and it has been attributed to critical slowing down of the order parameter fluctuations close to the phase transition from the ordered state to the paramagnetic state. ${ }^{8,9}$ However, the dependence of the magnetization on cooling rate and the temperature region where it is observed in our simulations are both too large to be attributed to critical slowing down. We rather believe that already far away from any critical temperature during the FH process small spin clusters connected to the percolating cluster turn over creating a domain-type state but without destroying LRO still present in the remaining parts of the percolating cluster. A dependence of this process on heating rate is then expected since large energy barriers have to be overcome. That at very low temperatures no time dependence of the FH curves is ob- served since then the thermal energy is not sufficient to turn spin clusters, supports this explanation. Clearly, this domain-type state is different from the domain state without LRO reached through field cooling. Note that no dependence on heating rate is also observed for high temperatures showing that the observed effect clearly acts on a time scale large compared to microscopic times. Strong support of our view comes from an inspection of spin configurations during FH. As a typical example they are shown in Fig. 2 in one layer of the three-

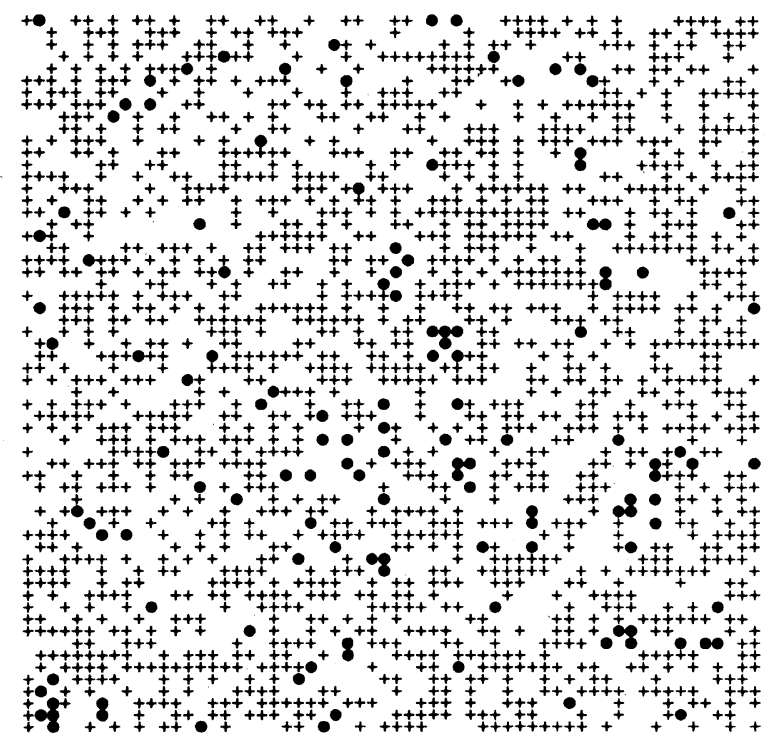

(a)

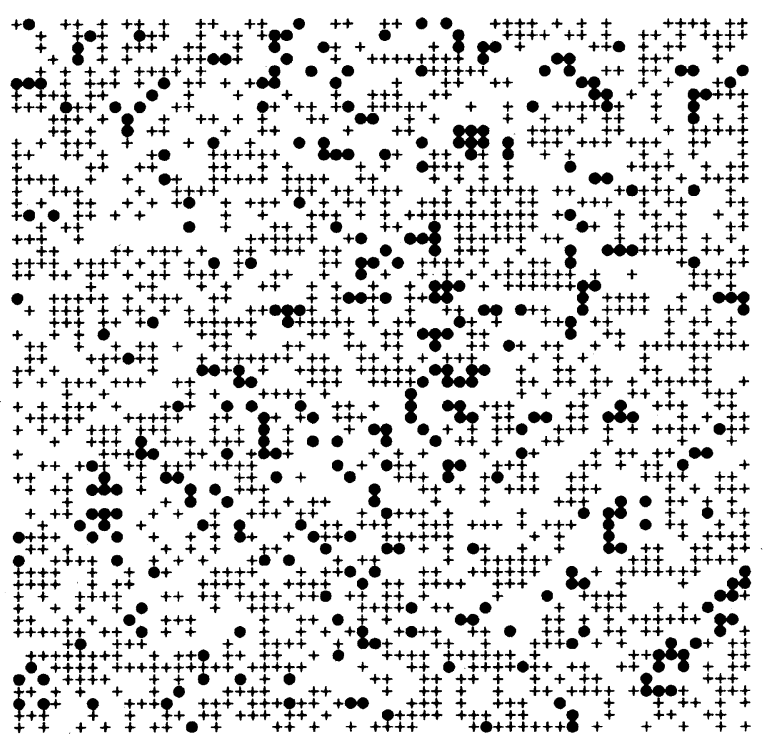

(b)

FIG. 2. Spin configuration in one layer of the $60 \times 60 \times 60$ lattice during $\mathrm{FH}$ for two different temperatures. (a) $T=0.8$; (b) $T=1.4$. Crosses and circles show the two staggered magnetizations. 
dimensional $60 \times 60 \times 60$ lattice for different temperatures. The circles and the crosses show the two directions of the staggered magnetization. Of course, some of the spins turned over are just thermal fluctuations. But since Fig. 2 shows only one layer of a three-dimensional system even single turned-over spins seen belong to a large extent to small clusters.

\section{RELAXATION BEHAVIOR OF THE REMANENT MAGNETIZATION}

We now turn to a discussion of the slow relaxation of the remanent FC magnetization observed here for the first time in Monte Carlo simulations. In principle the FC state could be obtained as described above by starting from the paramagnetic state which is then cooled down to low temperatures in a fixed field. The remanent magnetization and its relaxation is then obtained after switching off the field. This procedure has two disadvantages: first, for the cooling process we need a large amount of computer time so that we easily run out of time during the slow relaxation, which is really what we want to study; second, we want to study the relaxation process at different temperatures. If we cool down to these temperatures different initial states for the relaxation process are prepared making a comparison of the results rather difficult. Therefore, we used another strategy for the preparation of the domain state: we chose a fixed (small) temperature $T=0.4$ and a fixed field $H=2$ and performed $1000 \mathrm{MCS} /$ spin starting from a completely random spin configuration. Following the spin configurations in time it was observed that already after a few MCS/spin a state with domains and large magnetization grew out of the completely random state. This state relaxes in field towards a state very similar to the corresponding FC state obtained after cooling from high temperatures. During this slow relaxation the magnetization drops by roughly $20 \%$. The domain state so prepared serves as the initial spin configuration in the simulations of the time dependence of the remanent magnetization. These calculations were done by letting $H=0$ and choosing different temperatures $T=0.4,0.6,0.8$, and 1.0 . The investigation of the relaxation behavior at different temperatures starting from the same domain state has the advantage that the magnetization only depends on the relaxation processes, not on the different initial magnetizations.

In Fig. 3 is shown the spin configuration of the initial domain state in one layer of the three-dimensional $60 \times 60 \times 60$ lattice. Again the circles and the crosses show the two directions of the staggered magnetization. It is obvious from this figure that domain walls appear predominantly in regions with many vacancies.

The domain state shown in Fig. 3 has a finite magnetization which relaxes after turning off the external field. In Fig. 4 is shown the time dependence of this remanent magnetization for different temperatures. Note that the magnetization per spin of the initial domain state is around 0.3 . After switching off the field this magnetization drops by an order of magnitude within a few $\mathrm{MCS} / \mathrm{spin}$ before the slow relaxation process begins. The magnetization shown in Fig. 4 is an averaged quantity,

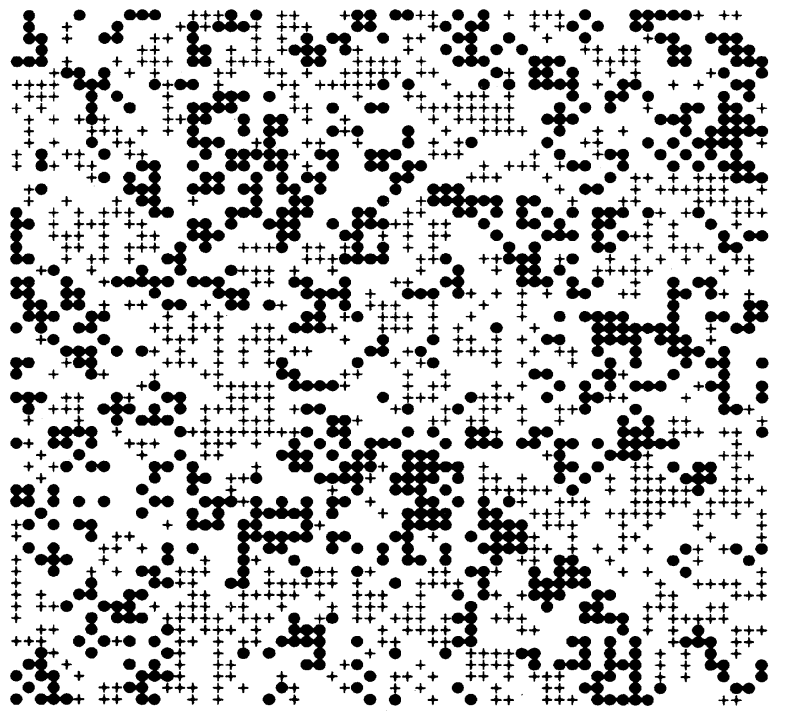

FIG. 3. Spin configuration of the domain state prepared as explained in the text. Crosses and circles show the two staggered magnetizations.

averaged over four randomly chosen configurations of vacancies. In addition, each data point shown is averaged over 20 consecutive MCS/spin.

In Fig. 5(a) is shown the "final" domain state, i.e., the domain state near the end of the simulation, for $T=0.4$. Comparing with Fig. 3 it is clearly seen that a readjustment of spins has taken place mainly in the domain walls, the volume part of the domains does not change supporting the ideas of Nattermann and Vilfan. ${ }^{4} \mathrm{~A}$ completely different situation is met for high temperatures where large volume relaxation takes place as can be sen in Fig. 5(b) which shows the final domain state for $T=1$.

Further evidence for the low-temperature mechanism proposed by Nattermann and Vilfan ${ }^{4}$ comes from a detailed study of the spin configurations close to a domain boundary. Figure 6 shows a small but typical part of the

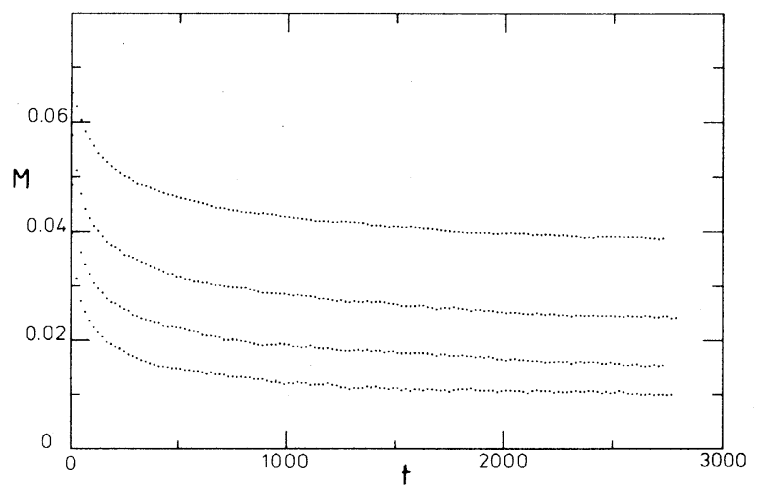

FIG. 4. Decay of the remanent magnetization $M$ after turning off the field for different values of reduced temperature $T=0.4$ (upper curve), 0.6, 0.8, and 1.0. Time $t$ is measured in MCS/spin. 
lattice. Here, the circles denote down-spins while the crosses are up-spins. Figure 6(a) shows the initial spin configuration prepared in a field which favors up-spins while Fig. 6(b) shows the final spin configuration. As can be seen in Fig. 6(a) in the initial configuration the domain wall-shown as a solid line-passes only through pairs of up-spins. We have checked this on a great number of domain walls and have found practically no exception to this rule. After switching off the field, pairs of up-spins are no longer favored. Therefore, spins along the domain wall readjust leading to small shifts of the walls in such a way that the number of neighboring spin-spin pairs cut

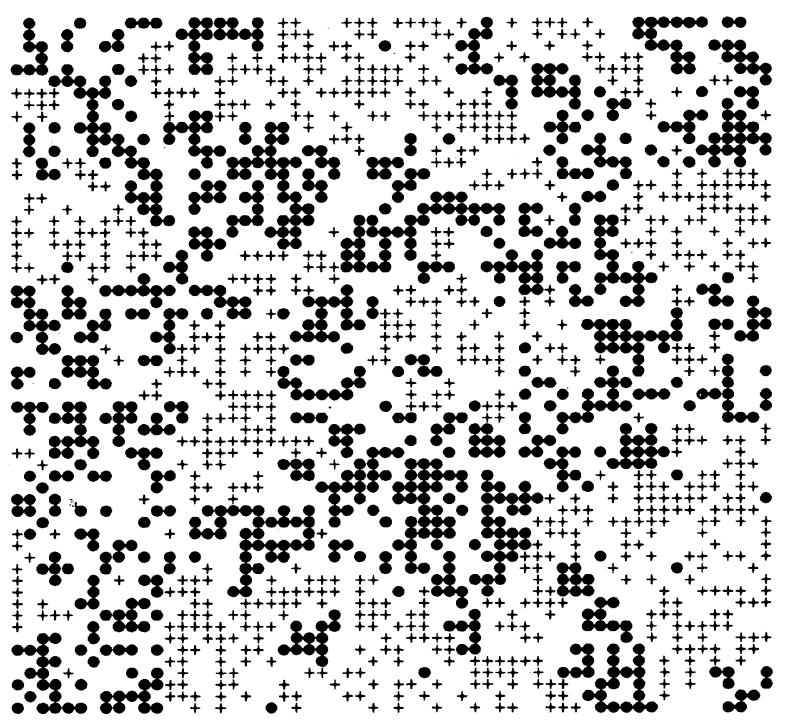

(a)

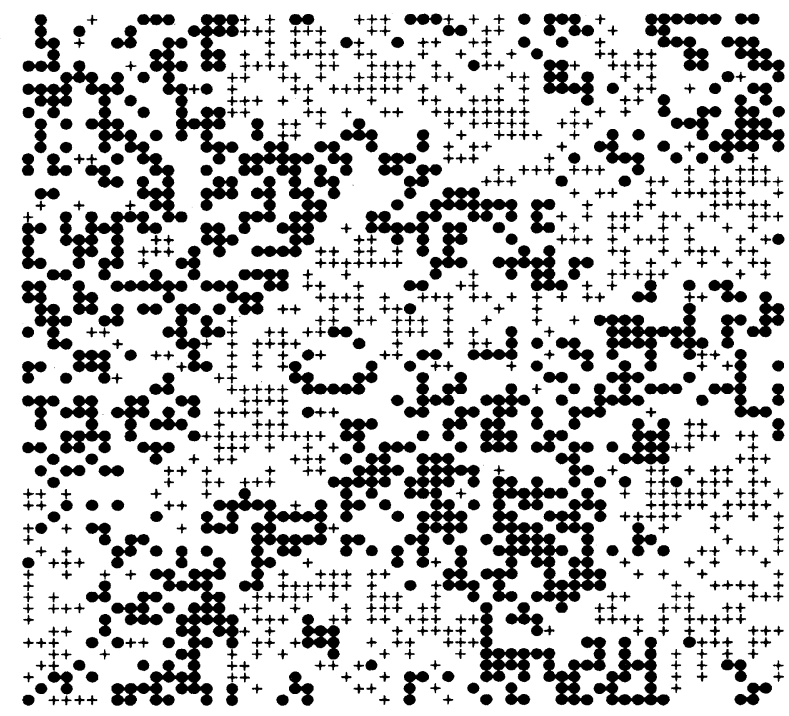

(b)

FIG. 5. Domain state after relaxation at different temperatures starting from the state shown in Fig. 3: (a) $T=0.4$; (b) $T=1.0$. Relaxation time was about $3000 \mathrm{MCS} / \mathrm{spin}$.

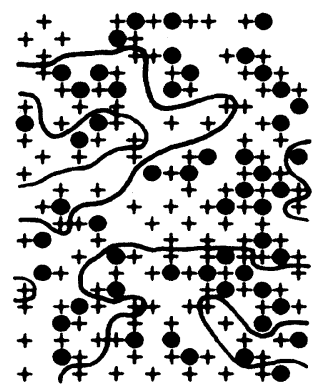

(a)

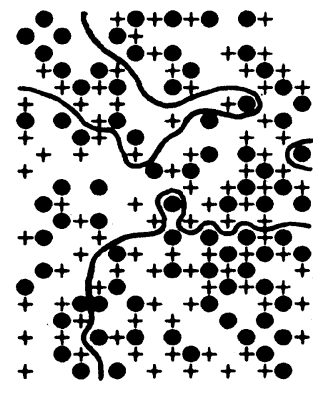

(b)
FIG. 6. Domain wall (solid line) in a small part of the lattice. (a) in the initial state, cf. Fig. 3. (b) in the final state, cf. Fig. 5(a). Crosses denote up-spins, favored by the external field, solid circles down-spins. $T=0.4$.

by the wall is drastically reduced, resulting in a reduction of surface energy. A related effect, a readjustment of domain walls seeking deeper random-field wells in a quenched field configuration, has been observed in Monte Carlo simulations of RFIM by Cambier and Nauenberg. ${ }^{15}$

We have tried to analyze our magnetization data using the relation

$$
M=C\left(\ln \frac{t}{\tau}\right)^{-\Gamma}
$$

for the time-dependent remanence proposed by Nattermann and Vilfan. ${ }^{4}$ In Eq. (1), $C$ is expected to be proportional to $T^{-1}, \tau$ is a microscopic time, while for $\Gamma$ the authors derive 0.4. A time-independent volume term expected to be small compared to the domain-wall contribution has been omitted. In Fig. $7 \ln M$ is plotted versus

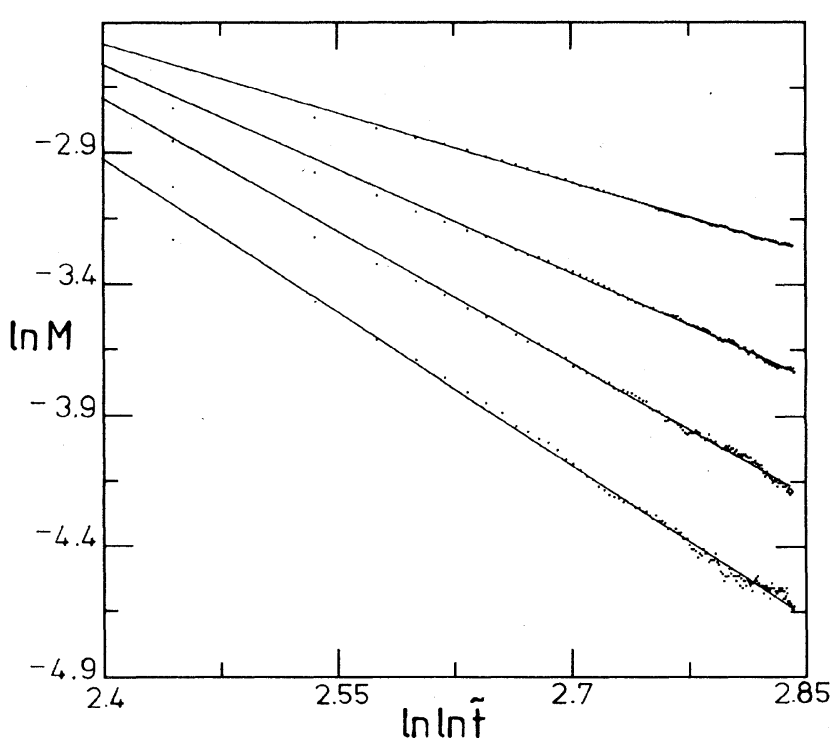

FIG. 7. $\ln M$ vs $\ln (\ln \tilde{t}) ; \tilde{t}=t / \tau ; \tau=1.02 \times 10^{-4}$. The solid lines are best fitted. 
$\ln [\ln (t / \tau)]$ showing that the data can fairly well be fitted to a straight line. Since $\tau$ is supposed to be a microscopic time we used for the four curves in Fig. 4 the same $\tau=1.02 \times 10^{-4}$ which was obtained from a least-squares fit of the low-temperature data for $T=0.4$ to Eq. (1). The values for $\Gamma$ are $1.8(T=0.4), 2.6(T=0.6), 3.4(T=0.8)$, and $3.9(T=1.0)$, much higher than expected. ${ }^{4}$ It is tempting to relate their increase with temperature to a crossover from domain-wall relaxation to domain growth where at least in the RFIM studied by Villain ${ }^{16}$ Eq. (1) is also valid but with a higher value of $\Gamma=1.5$. Unfortunately, the $\Gamma$ values obtained are even larger than this value. The expected $T$ dependence of $C$ could also not be confirmed.

As was discussed above, an increase in temperature leads to an increase in volume relaxation, and the simple law, Eq. (1), is no longer expected to be correct. Therefore, we also analyzed our data assuming power-law decay,

$$
M=C t^{-b} .
$$

Such power-law decay has been observed for the remanent magnetization in spin glasses. ${ }^{17}$ Figure 8 shows a log-log plot of the data. The values obtained for the exponent $b$ are $0.11(T=0.4), 0.17 \quad(T=0.6), 0.22$ ( $T=0.8$ ), and $0.25(T=1.0)$. The exponent $b$ appears to be nearly proportional to the temperature, a relation also observed in the spin glasses. ${ }^{17} C$ was found to be practically independent of temperature. In comparing the mean-square deviations of both fits we found that the low-temperature data $(T=0.4)$ were slightly better fitted by Eq. (1) while the opposite was found for all the other data. Significantly higher mean-square deviations for all temperatures were found by fitting the data to any stretched exponential. Therefore, the precise form of the time-dependent remanence remains open; more simulation work is needed to settle this problem.

Finally, some remarks concerning the values of $N, t$, and $H$ are in order. To study domain relaxation the system should be so large that it can contain many domains. Therefore, the lattice size was made as large as possible. A drawback of the rather large systems we used is that we had only a rather restricted simulation time of about $3000 \mathrm{MCS} / \mathrm{spin}$ for a single run. This restriction allows meaningful simulations only for temperatures $T \geq 0.4$ since for decreasing temperatures the dynamics of the system gets extremely slow. The domain size also depends on magnetic field. Increasing the field makes the domains shrink. As in most Monte Carlo simulations we have used values of the external field which are of the or-

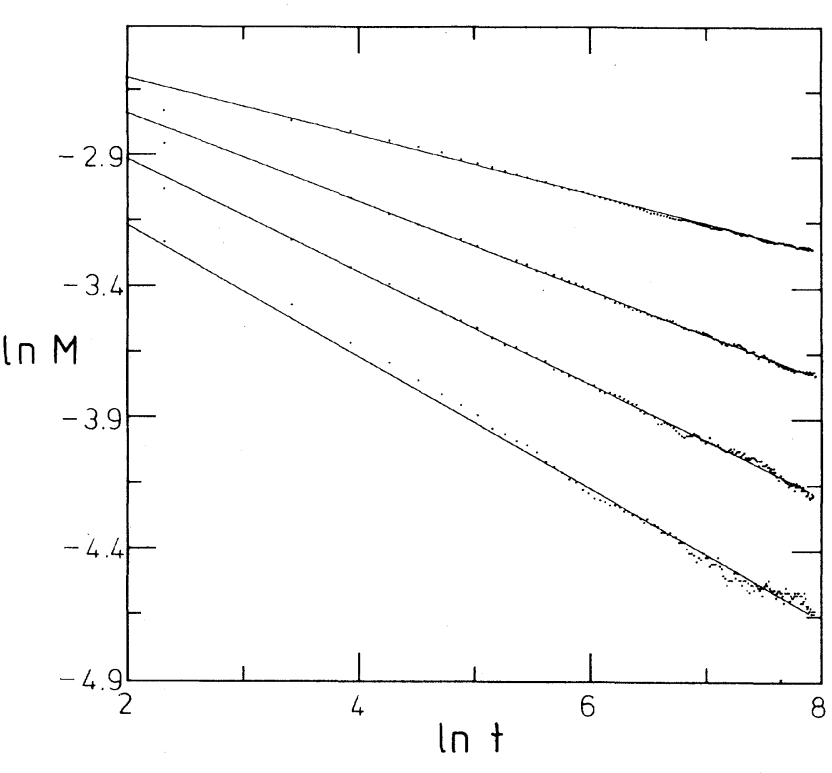

FIG. 8. $\ln M$ vs $\ln t$ for different values of reduced temperature: $T=0.4$ (upper curve), 0.6, 0.8, and 1.0. Solid lines are best-fits to the data.

der of the exchange interaction while usually the fields applied experimentally are much smaller. However, together with others we hope and believe that the domain properties studied in such large fields are also relevant to experiments.

\section{CONCLUSIONS}

The Monte Carlo simulations performed on large-scale lattices show the importance of domain pinning on the relaxation behavior in DAFF. Domain-type states appear during field cooling and $\mathrm{FH}$ but in the latter case without destroying LRO for not too high temperatures. Domains are strongly pinned to regions with many vacancies. For low temperatures relaxation takes place primarily within domain walls while for higher temperatures volume relaxation is also seen. The relaxation processes are connected with thermally activated jumps over large energy barriers leading to a number of irreversibilities.

\section{ACKNOWLEDGMENT}

It is a pleasure to thank W. Kleemann and U. Leitão for many discussions, especially on the experimental aspects of this work.
${ }^{1}$ For reviews, see R. J. Birgeneau, R. A. Cowley, G. Shirane, and H. Yoshizawa, J. Stat. Phys. 34, 817 (1984); D. P. Belanger, S. M. Rezende, A. R. King, and V. Jaccarino, J. Appl. Phys. 57, 3294 (1985); R. A. Cowley, R. J. Birgeneau, and G. Shirane, Physica 140A, 285 (1986).

${ }^{2}$ S. Fishman and A. Aharony, J. Phys. C 12, L729 (1979).

${ }^{3}$ A. Aharony, Europhys. Lett. 1, 617 (1986).
${ }^{4}$ T. Nattermann and I. Vilfan, Phys. Rev. Lett. 61, 223 (1988).

${ }^{5}$ H. Yoshizawa and D. P. Belanger, Phys. Rev. B 30, 5220 (1984).

${ }^{6}$ G. S. Grest, C. M. Soukoulis, and K. Levin, Phys. Rev. B 33, 7659 (1986).

${ }^{7}$ Ch. Ro, G. S. Grest, C. M. Soukoulis, and K. Levin, Phys. Rev. B 31, 1682 (1985). 
${ }^{8}$ U. A. Leitão, W. Kleemann, and I. B. Ferreira, Phys. Rev. B 38, 4765 (1988).

${ }^{9}$ P. Pollak, W. Kleemann, and D. P. Belanger, Phys. Rev. B 38, 4773 (1988).

${ }^{10}$ D. Chowdhury and D. Stauffer, J. Stat. Phys. 44, 203 (1986).

${ }^{11}$ U. A. Leitão and W. Kleemann, Phys. Rev. B 35, 8696 (1987).

${ }^{12}$ Y. Imry and S. Ma, Phys. Rev. Lett. 35, 1399 (1975).

${ }_{13}$ J. Z. Imbrie, Phys. Rev. Lett. 53, 1747 (1984).
${ }^{14}$ J. Bricmont and A. Kupiainen, Phys. Rev. Lett. 59, 1829 (1987).

15J. L. Cambier and M. Nauenberg, Phys. Rev. B 34, 7998 (1986).

16J. Villain, Phys. Rev. Lett. 52, 1543 (1984).

${ }^{17}$ J. Ferré, J. Rajchenbach, and H. Maletta, J. Appl. Phys. 52, 1697 (1981). 